\section{Research Square}

Preprints are preliminary reports that have not undergone peer review.

They should not be considered conclusive, used to inform clinical practice, or referenced by the media as validated information.

\title{
Parent Educational Intervention Program for Improving Parental Knowledge, Self-efficacy and Health Related Quality of life in Children with Sickle Cell Disease Using Smartphone Technology: A Randomized Controlled Trial
}

YUSRA AL NASIRI ( $\square$ yusra444@hotmail.com )

Ministry of Health Oman https://orcid.org/0000-0002-3993-9010

Eufemia Jacob

University of California Los Angeles

Eunice Lee

University of California Los Angeles

Adey Nyamathi

University of California Irvine

Mary-Lynn Brecht

University of California Los Angeles

Wendie A Robbins

University of California Los Angeles

Adhra Al Mawali

Ministry of Health Oman

Research

Keywords: Educational intervention, SCD, children, parents, smartphone technology

Posted Date: January 13th, 2020

DOI: https://doi.org/10.21203/rs.2.20544/v1

License: @ (i) This work is licensed under a Creative Commons Attribution 4.0 International License. Read Full License 


\section{Abstract}

Background

Sickle cell disease (SCD) is a genetic blood disorder that increases the risk for recurrent painful episodes. Parents' knowledge regarding SCD management is poor, leading to poor symptom management and lower Health Related Quality of Life (HRQOL) in children with SCD. The purpose of this study was to examine the effects of a parent educational intervention program ( PEIP) on the parental knowledge, self-efficacy and perception of the HRQOL of their children with SCD.

Methods

Two groups of Omani parents of children with SCD were randomly assigned to either an experimental group ( $n=37$ ) receiving PEIP accessed on a smartphone + weekly phone reinforcement for four weeks, or a control group $(n=35)$ receiving Standard Educational Program (SEP) as part of standard of care. Outcome measures were knowledge, self-efficacy and HRQOL of children with SCD. Knowledge Questionnaire, Self-Efficacy Scale, and HRQOL-SCD + HRQOL-GENERIC were administered twice (at enrollment, and 4 weeks after enrollment). Statistical Pakage for Social Science, version 24 was used for data analyses.

Results

Parents' knowledge and self-efficacy scores were significantly higher for the intervention group (PEIP) when compared to the SEP 4 weeks post intervention. Also, The total HRQOL scores were higher at 4 weeks compared to baseline, and were also higher in the PEIP compared to the SEP. Parents' knowledge and self-efficacy were significant predictors of HRQOL in children with SCD.

Conclusions

PEIP delivered by using a smartphone was effective in improving the parents' knowledge, self-efficacy in symptom management, and parent and child perception of HRQOL. PEIP was innovative in that it targeted all dimensions of HRQOL in children with SCD. Finally, the family played an important role in the process of care and therefore, developing family-based interventions is the key factor for improving HRQOL in children with SCD. The study highlighted the effectiveness of smartphone technology for delivering a high quality educational intervention program for parents and their families.

\section{Background}

Sickle Cell Disease (SCD) is a chronic, inherited hematological disorder that is associated with life- threatening complications that affect all major systems 2,6,8,22,27, 39, 55,59 It is characterized by crescent-shaped red blood cells that block the circulation of blood to tissues, resulting in tissue hypoxia. ${ }^{16,17,29,32,36,59}$ The most common genotypes of SCD are hemoglobin SS (HgbSS), hemoglobin SC (HgbSC), hemoglobin S beta thalassemia (HgbS $\beta$ ). Hemoglobin SS is the most severe (National Heart, Lung, and Blood Institute. ${ }^{40}$

The SCD Association of America estimates that approximately 70,000 to 100,000 individuals in the United States have SCD and 3 million have sickle cell trait. 52 SCD commonly occurs among individuals of African American decent. ${ }^{40,53}$ According to Centers for Disease Control and Prevention (CDC) statistics, approximately 1 of every 500 African Americans and 1 in 36,000 Hispanic Americans are born with SCD each year, and 1 in 13 African-Americans are born with sickle cell trait. ${ }^{18} \mathrm{SCD}$ in Oman is considered one of the most common genetic blood disorders, and contributes to increased mortality and morbidity rates in the country. ${ }^{38}$ It was reported that $6 \%$ of Omanis have SCD in 1995 survey. ${ }^{42}$ According to Oman Annual Health Statistics, the prevalence of SCD and other hematological disorders has increased from 86 to 141 cases per 10,000 Omanis from 1995 to 2005 due to high rate of consanguineous (first cousin) marriages. ${ }^{11}$ This was the only available data in Oman. The birth prevalence of symptomatic hemoglobinopathies in Oman was 1 in 323 , or 3.1 per 1000 live births; this rate included 2.7 per 1000 live births of HgbSS, with an estimate of 118 new cases per year. ${ }^{49}$

SCD leads to high mortality and morbidity rates in children 5 five years of age. ${ }^{58}$ Among the children with HgbSS, $1 \%$ die as a result of SCD-related complications during the first 3 years of life. ${ }^{18}$ In California and Illinois, the cumulative mortality rate was 1.5 per 100 African American children with SCD. The equivalent cumulative mortality rate for all African American infants born in California and Illinois was 2.0 per 100 African American newborns. ${ }^{18}$

Research studies that assessed HRQOL found that Children with SCD have poor HRQOL that affects the physical, emotional, social and cognitive. ${ }^{10,24,29,53,60}$ The corresponding interventions to address this problem are very limited. $1,3,4,7,15,60$ There is a dearth of literature about the strategies that improve HRQOL of Children with SCD. Few studies conducted educational interventions to improve the physical health of children with SCD. ${ }^{33,35,43,51}$ A quasi experimental study found that the caregiver's knowledge about $S C D$ and symptoms management were significantly increased after the intervention $(M=23, S D=3.6, p=.001)$ in comparison to pre intervention ( $M=16, S D=4.4)$. The rate of re-admission was significantly decreased two months after the educational intervention ( $M=2$, $S D=2.1, p<.05)$ compared to pre intervention $(M=4, S D=2.5)$. However, no control group was used, therefore, it was not clear whether the increase in knowledge was related to the intervention. There might be within group factors that led to the improvement in knowledge and may have produced biased results. ${ }^{51}$ Another study found that the parents' knowledge about the disease and ability to manage the symptoms at home were improved after a two-month period of using the written educational guide. More than (80\%) of the caregivers answered the questions correctly, (96\%) reported that the guide was easy to follow, and (96\%) reported that the guide was helpful. However, parents' knowledge was not measured at the baseline. Parents may have had a high knowledge before starting the intervention. Also, knowledge was assessed by asking the caregivers to respond to open-ended questions using paper and pen. The answers were analyzed subjectively by the researcher and this may suggest an intruding personal bias while interpreting the results. ${ }^{35}$ 
Two studies utilized a strategy that consisted of educational sessions and standardized pain medication orders for 6 months period. ${ }^{30,50}$ The intervention decreased the re-admission rate by (30\%) compared to previous year records and improved treatment adherence. ${ }^{30}$ The investigators reported that of a total sample of 100 children, only 30 children were re-admitted few months after the intervention. The main reason for hospitalization was the pain crisis (83\%).

The other 70 children experienced different symptoms of SCD; however, they did not require admission. ${ }^{30}$ Although health education was part of the intervention, no information was provided about the nature of the education, the frequency and the duration. Readmission rate was the primary outcome and parental knowledge was not measured. The study also did not have a control group, and therefore, it is not clear whether the decrease in readmission rate was related to the intervention. Similarly, the other study compared the readmission rate between the intervention group and the control group after a health education program. They found significantly lower readmission rates in the intervention group compared to the control group after 6 months period ( $M=2.1$, $M=2.3$, respectively, $p=.003$ ); however, the difference was not clinically meaningful, as the knowledge was not measured. No information was provided regarding the nature, the frequency, and duration of the health education program. Also, the control group was a retrospective cohort who was admitted the previous year for SCD complications, suggesting that both groups may not be similar at the entry level and there might be group variations between the two groups that could have affected the results. In addition, the knowledge was not measured in this study and the primary outcome was the readmission. ${ }^{50}$

A study used a computer program - SMART BRIGHT - to deliver educational materials to hospitalized children with SCD $(\mathrm{M}$ age $=11.7$ years, SD $=2.71)$. The study found a significant difference in the level of knowledge on disease related information between the experimental group $(M=8.73, S D=2.6)$ compared to the control group $(M=6.15, S D=3.21, p<.001$ ) before discharge from the hospital (Mean hospitalization $=5.1$ days, $S D=2.41, R a n g e=3-15$ days). $A$ significant difference was also found in the perceived peer social support $(M=15.1, S D=4.8 v s M=11.5, S D=4.5$, between the intervention and the control group respectively, $p<.05)$ and coping skills $(M=3.87, S D=0.35$ vs $M=3.61, S D=1.12$, intervention and control group, respectively, $p<.05)$. A convenience sample was used, which limits the generalizability of the findings. Also, the measurement of posttest was inconsistent across the participants. Many children had the posttest very early (less than 5 days post admission), which may have influenced the results. ${ }^{31}$

Self-efficacy is one's belief in the ability to execute behaviours necessary to attain specific performance. It reflects the individual's confidence in the ability to exert control over one's own motivation, and behaviour. ${ }^{9}$ Research on parental self-efficacy and the association between parental self-efficacy and perception of children's HRQOL in SCD are lacking. However, there are a few studies that evaluated self-efficacy on children with SCD, rather than parents. Self-efficacy in children was negatively associated with physical symptoms. The higher self-efficacy, the lower physical symptoms. ${ }^{19}$ Another study evaluated a guided imagery intervention on pain management in children with SCD. Those who were assigned to the guided imagery intervention reported higher self-efficacy following the training $(M=36.6, S D=3.9, p<.05)$ compared to pre-intervention $(M=26.4, S D=8.3)$. No studies were found that evaluated the parents' selfefficacy on their abilities to manage SCD and symptoms in children with SCD. ${ }^{23}$

Parents reported lower perception of HRQOL of their children compared to the children's own perception. Studies found that there was a significant difference between children's self-report $(M=88.69, S D=9.96)$ and parent-proxy reports $(M=85.51, S D=9.45, p<.001)$ of HRQOL in children less than seven years old. 20,21, 47 The parents' level of education was a significant predictor of parent's perception of HRQOL of children with SCD; parent's with high level of education, had better perception of their children's' HRQOL. ${ }^{20,46,53}$ One study reported that parents' educational level was a significant predictor of emergency room visits. Parents with high level of education were more likely to bring their children to emergency room for pain management $(F=1.29, p<.05)$ than their counterparts. 35

Interventions to improve HRQOL are lacking in SCD. Little is known about the impact of poor disease and symptom management on physical, emotional, and the cognitive aspects of HRQOL. There were no studies targeting parents as mediators to improve all aspects of HRQOL of children with SCD. It is not known whether parental knowledge and parental self-efficacy have effects on the HRQOL of children with SCD. Therefore, the purpose of the study was to examine the effects of a parent educational intervention program (PEIP) on the parents' knowledge, self-efficacy to manage symptoms at home, and parents' perception of the HRQOL of children with SCD in Oman.

\section{Specific Aims \& Hypotheses}

The specific aims and hypotheses of the study were:

1. To evaluate the effects of PEIP on parental knowledge and self-efficacy.

Hypothesis 1.1: Parents in the PEIP group would have higher scores on the SCD Parental Knowledge Questionnaire compared to the scores of parents on SEP group at 4 weeks post baseline.

Hypothesis 1.2: Parents in the PEIP group would have higher scores on the SCD Parental Knowledge Questionnaire in the posttest compared to baseline. Hypothesis 1.3: Parents in the PEIP group would have higher scores on Parental Self-Efficacy compared to the scores of parents on SEP group at 4 weeks post baseline.

Hypothesis 1.4: Parents in the PEIP group would have higher scores on the SCD Parental Self-Efficacy in the posttest compared to baseline.

2. To examine the effects of PEIP on parents' perception of HRQOL in children with SCD.

Hypothesis 2.1: Parents in the PEIP group would have higher scores on their perception of the child's HRQOL compared to the scores of parents on SEP group at 4 weeks.

Hypothesis 2.2: Parents in the PEIP group would have higher scores on the HRQOL scales in the posttest compared to baseline.

\section{Theoretical Framework}

The Health Related Quality of Life (HRQOL) theoretical framework as proposed by Wilson and Clearly was used to guide the content for the development of the Parent Educational Intervention Program (PEIP) and the Social Cognitive Learning Theory was used to explain the relationship between parental

Page 3/15 
knowledge, self-efficacy, and perception of child's HRQOL. ${ }^{13,57}$ The four constructs affect the individual's HRQOL, which is the extent to which an individual is happy in life as a whole. In addition, characteristics of the individual as well as the environmental factors are included in the model as nonspecific predictive variables of symptom status, functional status, general health perceptions and overall quality of life. ${ }^{26,54,57}$

The impact of PEIP on parents' self-efficacy and perceived HRQOL can be linked to the Social-Cognitive Learning Theory. ${ }^{13}$ In Bandura's theory human functioning is viewed as reciprocal interactions among behaviours, the environmental variables, cognition, and other personal factors. An important construct in Bandura's theory is perceived self-efficacy, which is the belief concerning one's capabilities to organize and implement actions necessary to attain designated performance level. Based on this model, self-efficacy has two components: efficacy expectation and outcome expectation. An increase in self efficacy leads to changes in performance, reduction in anxiety, and change in behavior. In the proposed study, improving parents' knowledge through the Parent Educational Intervention Program would lead to increased self-efficacy, which would consequently lead to improvement in pain and symptom management for their child. Bandura's theory suggests reciprocity in which more confident parents will lead to an increase in their ability to manage SCD, treatments, symptoms, and complications. Improvement in management of physical (pain, symptoms) and psychological (fear, worry, anxiety) symptoms will lead to improvement in HRQOL for the child.

The HRQOL framework suggests that the individual and environmental characteristics are the broader factors that directly influence the four constructs (biological function, symptom status, functional status, general health perceptions) and the construct of HRQOL (Figure 1). Since parents are an important part of the social environment surrounding the child, targeting the environmental factor (parents) and designing an educational intervention targeted towards parents will influence the child's overall HRQOL. The knowledge gained by parents from the Parent Educational Intervention Program is represented in the environment characteristics (Figure 1). The PEIP is designed to increase parental knowledge on 1) biological and physiological function, 2) symptom status, 3) functional status, and 4) general health perception of HRQOL. The improvement in the knowledge would increase parents' self efficacy (Shahine et al., 2015). Self-efficacy is considered the mediator that will facilitate parental learning and applying the knowledge as they manage SCD symptoms at home (Bandura, 1986).

\section{Methods}

\section{Design}

The study was a randomized controlled trial (RCT) with two groups of parents of children with SCD (Figure 5). One group was randomly assigned to the PEIP group, and the other was the control (SEP) group. The Parent Educational Intervention Program (PEIP)consisted of two video clips; the first video addressed the physical aspects - basic information about SCD, signs and symptoms, complications, potential triggering factors for sickle cell crisis, and the management of SCD (7 minutes). The second video (6 minutes) addressed 1) emotional aspects - fear, anxiety, worry, sleep disruptions; 2 ) social relationship with peers, siblings, ability to enjoy leisurely activities; and 3) cognitive aspects - the child's ability to do well in school, ability to communicate, solve problems, make decisions, resolve conflicts. The PEIP was delivered via smartphone with reinforcement phone conversation facilitated by the PI once per week, over a 4-week period. Measurement of outcomes (parents' knowledge, parents' self-efficacy, and parent's perceptions of HRQOL as well as the children's perception of their HRQOL were done at baseline, and at the end of the intervention, which was 4 weeks after enrollment.

\section{Setting}

The parents (either father or mother) of children with SCD were recruited from the Royal Hospital (RH) and Sultan Qaboos University Hospital (SQUH), both tertiary hospitals in the Sultanate of Oman. All parents of children with SCD who were followed in the hematology clinics in these hospitals were invited to participate. Approximately, 300 children with SCD were seen annually in the hematology clinics at both hospitals.

\section{Sample}

A convenience sampling approach was used to recruit parents of children with SCD. First, parents were eligible if the age of their child with SCD is between 8 to 12 years. In Oman, children until 12 years of age were seen in the pediatric clinics, and children ages of 13 and older were seen in the adult clinics. The rationale for selecting this age group was because school age children were able to report pain, and other symptoms related to the disease. Also, the information on the emotional, social, and cognitive health were more important for children in this age group. An assumption of the study was that parents, as the child's primary caregiver, would be able to apply the knowledge as they provide care for their child.

Second, the parents were eligible if they were Omani citizens because the PEIP was specifically designed so that it was culturally appropriate for Omanis. Therefore, parents from other nationalities, who did not speak Arabic and who came from different cultures were excluded. Parents were also excluded if they: 1) were not available for consenting procedures; 2) were not willing to participate in completing the study procedures; and 3) had physical, cognitive, and/or neurological impairments that prevented them from consenting and/or completing study procedures. The nurse screened the eligible participants for any neurological impairment using the Decision-Making Capacity Assessment tool. This was done to assess the ability of parents who may have or may be experiencing cognitive impairments to make an informed decision about being part of the study. In the event that the Omani parents were not able to read, a nurse not involved in the study procedures would read the study materials.

The PEIP was delivered via a smartphone. Parents who did not have smartphones were included, and were provided with a smartphone for study use. A total of nine parents were excluded from the study as four of them were not Omanis and the other five were not willing to participate in completing the study procedures.

\section{Sample Size determination}


The total number of parents that were enrolled were 74 (37 in each group). Using G-power, the sample size of 37 in each group was able to detect a 0.71 effect size increase in knowledge ${ }^{51} 0.59$ increase in parent's self-efficacy ${ }^{23}$ and 0.5 increase in HRQOL ${ }^{41}$ compared to control groups.

A trained nurse coordinator worked with the hematology team at the Royal Hospital identify the eligible parents, screened the prospective participants provided information about the study for eligible participants, and created the list of children's names and their parents, who were interested; the list was provided to the principal investigator (PI) for more detailed explanation of the study and consenting procedures. In the event that a parent was not able to read, the nurse coordinator explained in terms the parent is able to understand about the study.

The PI met parents assigned to the intervention group in a private room in the clinic for 15 minutes to explain the details of the study procedure to the parents and their children using the study information sheet. They were allowed to ask questions, and talk with family members and care providers as needed. Parents signed the consent, and the child signed the assent form prior to enrollment and study procedures.

Randomization Procedures: To minimize the risk of contamination, a cluster randomization approach was used, with enrollment occurring on two separate days for the intervention (PEIP) and the control groups (SEP). To minimize potential temporal bias, a coin flipping was used every week to decide on the days for the cluster placement to the PEIP or the SEP group. Participants enrolled during the first day were assigned to the PEIP group and those attending the clinic on the subsequent day were assigned to SEP; this procedure was continued alternatingly.

Data Collection Procedures. All parents, regardless of group assignment were asked to complete the following pre-and post-intervention assessments using paper and pen at time of enrollment: 1) Parents Knowledge Questionnaire (PKQ) (10 minutes), 2) PedsQL (Generic) (5 minutes), 3), PedsQL SCD module (10 minutes), 4) Self-Efficacy Scale (SES) (5 minutes), 5) Demographics Questionnaire (2 minutes). The children were asked to complete the following age appropriate PedsQL: 1) PedsQL (Generic) (5 minutes), 2) PedsQL SCD module (5 minutes). Completion of the assessments took approximately 30 minutes at the beginning and at the end of the intervention period.

Parental Knowledge Questionnaire (PKQ). The SCD Parental Knowledge Questionnaire (PKQ) has 25 items; 16 items were True/False statementsand 9 items were multiple-choice (A, B, C, D) type questions. The items measured the knowledge about SCD, signs and symptoms, complications, treatment and triggering factors (16 items). The other nine items were psychological (4 items), social (3 items), and the cognitive problems (2 items) (derived from NHLBI, 2014). . Each item on the PKQ was scored as 1 to yield a maximum total score of 25 points; , range 0 to 25 , with higher scores indicating higher knowledge. The English to Arabic translation was available, and the Arabic back to English translation procedure as well as the content validation were done by 3 experts ( 2 hematologists, one nurse in Oman) was done prior to implementation. The readability index for PKQ is appropriate for the $7^{\text {th }}$ grade level.. The Arabic PKQ was then piloted on 5 parents who were excluded from the study. The internal consistency reliability using Cronbach alpha was (0.80) prior to commencement of the study and (0.87) 4 weeks post intervention. .

Pediatric Quality of Life Inventory (PedsQL-GENERIC). The Pediatric Quality of Life Inventory (PedsQL-GENERIC) developed by Varni and colleagues was a generic core scale that had 23 items ara designed to measure four dimensions of HRQOL: 1$)$ physical functioning (8 items), 2) emotional functioning (5 items), 3) social functioning (5 items) and 4) school functioning (5 items). The parent proxy report format and the corresponding child version for $8-12$ years was used. ${ }^{56}$ The English to Arabic and Arabic back to English translation, as well as the content validation procedures done by 3 experts ( 2 hematologists, one nurse in Oman) prior to implementation. Both parent and child versions of the PEdsQL-GENERIC was then piloted on 5 parents and children who were excluded from the study. The internal consistency reliability using Cronbach alpha was (0.8) prior the commencement of the study and (0.85) 4 weeks post intervention. The internal consistency reliability of the Arabic version is (0.80).

Scoring \& Interpretation of PedsQL-GENERIC. The items on the PedsQL-GENERIC were scored from 0-4 scale, and rated as 0 is "never a problem"; 1 is "almost never a problem"; 2 is "sometimes a problem", 3 is "often a problem"; and 4 is "almost always a problem". Following the instructions for scoring, the 0 to 4 scores were reversely converted to the 0 to 100 scores for standardized interpretation, so that 0 was scored as $100 ; 1$ was scored as $75 ; 2$ was scored as $50 ; 3$ was scored as 25 and 4 was scored as 0 . The items were averaged so that the total scores ranged from 0 to 100 and the $t$ the Physical and Psychosocial Summary Scores were computed. Higher scores indicated higher HRQOL. ${ }^{56}$

\section{Pediatric Quality of Life Inventory SCD (PedsQL-SCD)}

The HRQOL-SCD module had 43 items, and nine scales: Pain \& hurts (9 items), pain impact (10 items), pain management \& control (2 items), worry I (5 items), worry II ( 2 items), emotion ( 2 items), treatment (7 items), communication I ( 3 items), and communication II ( 3 items). ${ }^{48}$ Similar to the parent version, it has a 5 point Likert type response scale. The English to Arabic and Arabic back to English translation, as well as validation procedures of both parent and child versions were done by 3 experts ( 2 hematologists, one nurse in Oman) prior to implementation. Both parent and child versions of the PedsQL-SCD were then piloted on 5 parents and children who were excluded from the study. The parent proxy report format and the child version for 8-12 years was used. Both parent and child versions of the PedsQL-SCD was then piloted on 5 parents and children who were excluded from the study. The internal consistency reliability showed Cronbach's coefficient alpha 0.70 for children, which was considered acceptable. Internal consistency reliability coefficients for the PedsQL-SCD Module showed reliable with Cronbach's alpha (0.93) for children with SCD. The PedsQL was piloted, and the internal consistency reliability was (0.85) prior conducting the study and (0.89) 4 weeks post intervention. Also, test-retest reliability, and estimating interclass correlation (ICC) for the tool was done. The period between the test-retest was 2 days. Two days was found appropriate because children with SCD were prone for any complication at any time; therefore, waiting for a longer period to conduct the retest may result in scores variation. The internal consistency reliability was (0.9).

Scoring \& Interpretation of PedsQL. Similar to the HRQOL-GENERIC, the items on the HRQOL-SCD Module was scored from 0-4 scale, 0 was "never a problem"; 2 was "almost never a problem"; 3 was "often a problem"; and 4 was "almost always a problem", and were converted to the 0 to 100 scores for 
standardized interpretation as described above. The items were averaged and the Physical and Psychosocial Summary Score were computed as described above (Panepinto et al., 2013).

\section{Parent SCD Self-Efficacy}

The self-efficacy scale for parents (SES) was adopted and modified from self-efficacy instrument specific to sickle cell disease (SCSES) that was developed by Edwards and colleagues for use in adults with sickle cell disease. The original questionnaire has 9 items that measured disease specific perception of selfefficacy regarding the patients' ability to function on a day-to-day basis and to manage their child's symptoms and pain related to SCD. ${ }^{24}$ The items in the parent self-efficacy tool remained the same. However, wording was changed so the items reflect that it was the parent reporting (rather that child reporting) of their ability to manage the symptoms, and pain of their children on a day-to day basis. The questionnaire was administered at the time of enrollment and 4 weeks post intervention. The internal consistency reliability showed a Cronbach's alpha of $(r=0.89)$. In the study, experts assessed the modified tool for content; construct validity before and after translation to Arabic languae. In addition, the SES was piloted on five participants who were excluded from the study, and the internal consistency reliability indicated the Cronbach's $a=0.9$ prior conducting the study, and $a=0.954$ weeks post intervention.

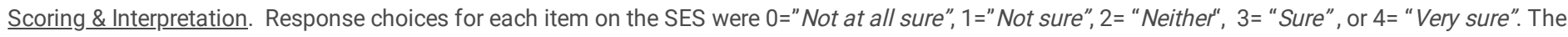
total scores were obtained by summing responses for all nine items; the range of scores were 0 to 36 , with higher scores indicating greater self-efficacy.

\section{Demographics \& Medical Information Sheet}

Parent demographics, which included age, gender, educational level, socio-economic status, and marital status were collected by the nurse coordinator in the haematology clinic at the time of enrolment. In addition, demographics and medical information related to the children (age, gender, use of hydroxyurea, SCD diagnosis, history of SCD-related complications, including number of pain crisis requiring hospitalization the previous year) were also collected.

Procedures for Delivery of Parent Educational Intervention Program (PEIP). Following the pre-intervention assessments, parents assigned to the PEIP group were oriented by the PI and requested to navigate the educational materials on their smartphones. The orientation session was 20 minutes. The two video educational materials were downloaded to the parents' own smartphone. If parents did not have one, parents were provided with one; however, all parents had smartphones and were not required to get another one. Downloading of the two videos into the smartphone took approximately 2-3 minutes depending on the network. The parents were allowed to practice accessing the materials and opening the videos.

After the orientation session, the PI informed the parents to watch both videos on their free time when they reached home and that the PI would contact them by a phone call once per week (every Wednesday), focusing on one section per week (physical, emotional, social, cognitive health) for four consecutive weeks, to remind parents to watch the videos, read the materials, and refer to the material about pain control. Parents were encouraged to refer to videos whenever needed.

Call duration was approximately 10- 12 minutes per participant. Most of the time, the parents picked up the phone call from the first time; however, some parents were approached after the second call. In the first 2 minutes of the call, the parents were asked to brief about the section they saw that week. This was done to ensure that parents have actually seen the assigned section and understood the information provided for them. Generally, all parents were able to provide information about the section they saw.

For the physical aspect, parents were asked about pain management for their child "what was done or what would be done" to minimize the child's pain and they were instructed to refer to the video for pain management. Also, they were asked to list the precautions they have taken to prevent future pain for their children, and they were referred to watch the video for VOC triggering factors. If the child was on hydroxyurea, the parents were encouraged to give it on time and as prescribed. For the emotional aspect, after 2 minutes briefing about the section, the parents were asked if they have noticed any emotional changes in their children and how they responded to them. The PI requested the parents to refer to the information on the role parents on improving the emotional health for their children. Similar to the emotional aspect, for the social aspect, the parents were asked about the social problems or changes observed in their children and how they responded to them. Parents were reminded to refer to the material on the parents' role in enhancing the child's social health. For the cognitive aspect, parents were asked about the child's current school performance, any cognitive issues, and what was done to solve those issues. The PI emphasized on the importance to collaborate with the school for better cognitive outcomes and refer to the material on the cognitive health.

In addition, all participants' questions and concerns were addressed during the call. Any problem experienced by the parents in regards to the video were also addressed. Almost all the parents watched the videos 4-5 times throughout the intervention. A unique feature of the $P E I P$ was the ability to allow parents the opportunity to openly talk about the child's health that were not routinely part of clinic visits, and reinforced different aspects of the HRQOL-physical, emotional, social, cognitive health respectively during the four weeks.

\section{Procedures for Delivery of Standard Education Program (SEP).}

To avoid contamination, the parents in the SEP were oriented separately in the Pediatric hematology clinic. The parents assigned to the SEP met with the PI for an orientation session at a private room in the hematology clinic. The PI explained the details of the study. Parents were allowed to ask questions, and talk with family members and care providers as needed. They were given time to think about participation as needed. The participants were informed that care would continue regardless of whether they participate in the study or not. They were also informed that the information would be kept confidential so that no one would know the individual responses to the questionnaires. Moreover, the parents were informed that the participation was voluntary and they had the right to withdraw anytime from the study. Parents received a $\$ 30$ "Lulu" a shopping voucher for their participation at the end of the study. Parents willing to participate were then asked to sign the consent form when they were ready. When consenting process finished, the parents were instructed to complete the study questionnaires, using paper and pen. 
The control group received the standard education program (SEP) that was given by the staff nurses in the clinic. The SEP consisted of verbal information about the follow up appointments. For the study, a booklet containing questions and answers related to typical questions and answers that parents receive about SCD were distributed to the control group. Examples of the questions were: "What diet should sickle cell patients follow? "What is the effect of cold temperature on people with SCD?" "How does warm temperature affect people with SCD?" "Why should I take the vitamin folate?" There were no videos accessible by smartphone, and no follow-up phone calls about their understanding of health education or allowing them to talk about the physical, emotional, social, and cognitive aspects of the child's health. To minimize the risk of contamination through information sharing between parents in the PEIP and the SEP groups, the researcher coordinated with the registration department and the assigned physicians to schedule separate dates for the parents in the PEIP and the SEP group for the subsequent appointments during the 4-week period of participation in the study.

\section{Results}

\section{Data Analyses}

The data were analyzed using SPSS (version 24). Frequencies, means, and standard deviations were calculated to describe the characteristics of the parent and child sample. Scores were calculated for primary outcomes. Repeated measures ANOVA were used to tests differences between the PEIP and SEP groups at baseline and 4 weeks. A p-value $(p \leq 0.05)$ was considered significant.

\section{Demographics}

\section{Parents' Demographics}

A total of 72 parent and child participants were enrolled; 37 in the PEIP group and 35 in the SEP group (Table 1). Parental age ranged between 28 and 55 years. There were more mothers in the $P E I P(\mathrm{n}=25 ; 68 \%)$ and compared to the $\operatorname{SEP}(\mathrm{n}=11 ; 31.4 \%)$. All parents $(100 \%)$ in the $S E P$ were married, compared to $95 \%$ of the parents in the PEIP. Parent educational level was equivalent in both groups with the majority completing a high school degree (64\% $P E I P, 75 \%$ SEP), and fewer with Associate, Bachelor or Master degrees (Table 1). About half (52\%) of the participants were residing in the northern region in Oman, mostly from Batinah (27\%) and Muscat (25\%). About one third (31\%) were from the west (Al Dhakilyah; $28 \%$ ) and central (Al-Dahira; $3 \%)$. Few were residing in the east $(17 \%)$, mostly from Sharqiyah. Two participants withdrew from the study; one participant had death in the family and the other participant missed the flight from Salalah (South of Oman) to Muscat on that day.

\section{Children's' Demographics}

Children's age ranged from 8 to 12 years. The sex distribution in the children's group was equivalent with 19 (51\%) males in the $P E I P$ and 15 (43\%) males in the SEP. Less than half $(41 \%$ PEIP, $49 \%$ SEP) of the children were receiving hydroxyurea, the medication for minimizing vaso-occlusive episodes in SCD. No significant age differences in parents and children between the PEIP and SEP groups.

\section{Parental Knowledge outcome}

Results indicated a significant group-by-time interaction $(F(1,66)=363.7, \mathrm{p}<.001)$ in knowledge scores (Figure 1$)$. The findings elicited a significant difference in change for the PEIP from baseline to 4 weeks posttest $(F(1,32)=23.14$, partial $\eta 2=.4, p<.001)$. Knowledge scores were significantly higher at 4 weeks $(21.8 \pm 1.3)$ for the PEIP compared to baseline $(11.00 \pm 2.5)$. In addition, the differential change across groups produced a significant difference in knowledge scores at 4 weeks $[F(1,66)=477.9, p<.001$, partial $\eta 2=.87]$. The PEIP group had significantly higher knowledge scores $(21.8 \pm 1.3)$ at week 4 , compared to the control group at 4 weeks $(11.7 \pm 2.3)$. Thus, results supported Hypotheses 1.1 and 1.2 .

\section{Self-Efficacy outcome}

There was a significant group-by-time interaction in the self-efficacy scores $[F(1,66)=790.02, p=.001]$ (Figure 2$)$. The findings revealed a significant difference in change for the PEIP from baseline to 4 weeks posttest $(F(1,32)=12.4$, partial $\eta 2=.3, p<.001)$. The PEIP had significantly higher self-efficacy scores $(30.2 \pm 2.3)$ at 4 weeks, compared to baseline $(13.7 \pm 2.5)$. In addition, the differential change across groups produced a significant difference in knowledge scores at 4 weeks $[F(1,66)=666.2$, partial $\eta 2=.91, p<.001]$. The $P E I P$ had significantly higher self-efficacy scores (30. $2 \pm 2.3)$, compared to SEP group at 4 weeks. $(15.1 \pm 2.2)$ Thus, results supported Hypotheses 1.3 and 1.4 .

\section{HRQOL-SCD outcome}

There was a significant group-by-time interaction in the health related quality of life (HRQOL-SCD) scores $[F(2,69)=187.9, p=.001]($ Figure 3$)$. The findings revealed a significant difference in change for the PEIP from baseline to 4 weeks post intervention. The PEIP had significantly higher HRQOL-SCD scores (76.2 $\pm 6.15)$ at 4 weeks, compared to baseline $(53.2 \pm 7.5),[F(1,32)=10.91, p=.002$, partial $\eta 2=.3]$. The differential change across groups also produced a significant difference in HRQOL-SCD scores at 4 weeks $F(1,66)=148.92, p=.001$, partial $\eta 2=.70$. The PEIP had significantly higher HRQOL-SCD scores $(76.2$ $\pm 6.2)$ at 4 weeks, compared to the SEP group $(57.6 \pm 8.3)$ at 4 weeks. Thus the results supported hypotheses 2.1, and 2.2.

\section{HRQOL-Generic outcome}

Similarly, the findings elicited a significant group-by-time interaction in the health related quality of life $(\mathrm{HRQOL}-\mathrm{Generic})$ scores $[F(1,70)=349.74, p=.001]$ (Figure 4). The PEIP had significantly higher HRQOL-Generic scores $(78.2 \pm 3.47)$ at 4 weeks, compared to baseline $(46.9 \pm 10.50),[F(1,32)=13.16, p=.001$, partial $\eta 2=.3]$. In addition, the differential change across groups produced a significant difference in HRQOL-Generic scores at 4 weeks $[F(1,66)=317.26, p=$ 
.001 , partial $\eta 2=.8]$. The PEIP had significantly higher HRQOL-Generic scores $(78.2 \pm 3.47)$ at 4 weeks, compared to the SEP group (50.5 \pm 10.96$)$ at 4 weeks. Thus the results supported hypotheses 2.3 , and 2.4 .

\section{Correlation Between Parent \& Child HRQOL Scores}

The parent and child HRQOL-SCD scores were moderately correlated $(r=.44)$ at baseline. The parent and child HRQOL-GENERIC scores were also moderately correlated (overall $r=.38, p=0.001$ ) at baseline (physical=.4, emotional=.3, social=.4, cognitive=.5). The correlations were higher at 4 weeks for both HRQOL$\operatorname{SCD}(r=.91)$ and HRQOL-GENERIC (overall $r=.95, p=0.001$ ) [physical $=.8$, emotional $=.9$, social $=.9$, cognitive $=.8$ ].

\section{Discussion}

\section{PEIP \& Parental Knowledge}

We compared the effects of two educational intervention programs, PEIP and SEP on parents' knowledge, self-efficacy, and perception of the child's HRQOL. There was significant improvement in the parents' knowledge after the $P E I P$, and their knowledge scores were significantly higher at week 4 when compared to the SEP at week 4. The findings are consistent with other reports indicating significant improvements in knowledge after educational intervention programs. ${ }^{7}$

It is important to note that $75 \%$ of the parents had low educational status (less than or equivalent to a high school education); yet, they were able to learn about the disease and symptom management from the PEIP, and applied their knowledge. Previous studies indicated that low educational status of parents was associated with adverse health outcomes in children. ${ }^{51}$ The PEIP was culturally-sensitive and used a simple language that could be understood at a $5^{\text {th }}$ grade reading level, and caregivers with low literacy level. ${ }^{51}$ Omani parents with low literacy status were able to understand the content. The cultural feature of the PEIP made it readily acceptable by the Omani parents since it reflected the Omani culture.

A unique feature of the PEIP was that it may be viewed by using a smartphone which was powerful and congruent with the increasing availability and use of technology in low resource settings, in relatively remote areas away from the Comprehensive Sickle Cell Center. Participants from the study represented several regions in the east, west, and northern Oman. Having the PEIP in the smartphones facilitated retention and having the ability to refer and access information about SCD as needed. Our study is the first to use this innovative innovative educational approach in using smart phones, for delivering important health information that is comprehensive, more engaging than the traditional educational methods, and may be readily accessible multiple times at home. ${ }^{34}$

Another unique feature of PEIP is that it facilitated communication with parents every week for 4 weeks to discuss the material, address questions and concerns about SCD, and discuss how the content may be individually applied as they provide care for their children with SCD. The weekly contact facilitated communications with parents and care provider as previously reported using smartphones. ${ }^{34}$ It is possible that the individual attention provided during the 4 week study period, may have empowered them, thereby increasing their self-efficacy.

\section{PEIP \& Self-Efficacy}

Our data showed that parent self-efficacy in PEIP was higher at 4 weeks compared to baseline, and was also higher compared to the SEP at four weeks. Findings from the study support the association between self-efficacy and individual capabilities. It is possible that with improvement in parents' knowledge through the PEIP, self-efficacy increased, which consequently lead to improvement in their ability to manage pain and symptoms for their child with SCD, as previously reported. ${ }^{35,37}$ Dobson and colleagues have previously demonstrated improvement in self-efficacy in children with SCD, with corresponding improvement in symptom management after educational interventions. ${ }^{23}$ Our study was the first to evaluate parents' self-efficacy of children with SCD.

\section{PEIP \& HRQOL}

Findings from the study indicated improvement in the physical dimension of HRQOL. One of the main content that was emphasized in the PEIP was the physical dimension, such as avoiding the triggering factors for the acute pain episodes, the most distressing complication of SCD. Specific instructions were provided for parents to avoid exposure to hot and cold weather, dehydration, performing excessive exercises, experiencing stress, and being exposed to infectious agents, and how to prevent them. The PEIP also described the role of hydroxyurea in reducing the frequency of the pain episodes, and encouraged adherence to hydroxyurea. Adherence is important because Badawy and colleagues found that a major barrier to adherence to hydroxyurea was poor understanding of the use and benefits of this medication. The PEIP allowed parents to learn more about hydroxyurea, and allowed them to express thoughts and concerns, and ask questions during the phone call, thereby promoting adherence to its administration. ${ }^{12}$

In Oman, the lack of parents' knowledge to manage symptoms of SCD at home, led to increase in healthcare utilization (clinic, ED visits) and hospitalization. Omani families typically manage pain using traditional practices such as herbs and oil massage. Providing medicine to alleviate pain is given late after trying non-pharmacological interventions. PEIP not only provided information to parents about hydroxyurea, but also specific instructions on how to assess child's level of pain, manage pain at home based on severity, minimize delay in pain treatments, and when to proceed to the Emergency Room to minimize serious complications. Therefore, the ability of the parents to recognize the triggers, assess and manage pain at home, and most importantly their prompt responses to the child's pain, most likely affected their children' physical health.

We found that at baseline, children reported lower scores on the physical aspects of HRQOL when compared with the parents' ratings, suggesting that parents' may not be aware of the child's pain experiences prior to the PEIP intervention. It is interesting that the correlation between the parents' and the children on the physical aspects of HRQOL was stronger at the end of 4 weeks. This finding suggests that parents were able to assess pain accurately and respond to pain more readily at 4 weeks. Children on hydroxyurea had higher HRQOL scores when compared to children not on hydroxyurea. It is possible that the PEIP 
increased adherence to hydroxyurea thereby decreasing frequency and improvement in parent management of the acute pain episodes. The findings are consistent with other reports suggesting improvement in the HRQOL of children with SCD using hydroxyurea drug. ${ }^{5,14,28,41}$

The total HRQOL scores were higher at 4 weeks compared to baseline, and were also higher in the PEIP compared to the SEP. The PEIP provided comprehensive information including specific information on the four important dimensions of HRQOL (physical, emotional, social, cognitive), since the PEIP addressed only the physical management of SCD, but also strategies for improving children's emotional, social, and cognitive health. The inclusions of strategies for improving the physical, emotional, social and cognitive status may have led to improvement in the overall HRQOL in children with SCD. Knowledge gained through the PEIP affected the parents' self-efficacy; which consequently improved their perception of the child's HRQOL, as our findings indicated that knowledge and self-efficacy were significant predictors for improving HRQOL.

\section{Limitations \& Recommendations}

There are several limitations that need to be considered when interpreting the findings in our study. First, the sample size was small and was conducted only in Oman; therefore, it is not possible to make generalizations to other settings. Second, we only included parents and children 8 to 12 years old, and therefore would not be generalizable to parents and children younger than 8 years, or older than 12 years. We were not able to examine the effects of PEIP on health care utilization (clinic, ER visits) and frequency and duration hospitalization during the 4 months before and 4 months after intervention. We also did not evaluate parents' satisfaction with the PEIP, and other features that they found most useful, not so useful, and potential barriers to future use. Finally, the duration of the intervention was only for 4 weeks, and its effects over a longer period of time were not evaluated. Future studies are therefore, recommended to evaluate the impact of PEIP on health care use, assess the features that were most and least useful, barriers to future implementation, determine whether the effects may be sustained beyond 4 weeks, and whether additional reinforcements may be required over a longer period of time. Cultural adaptation of the PEIP to other languages, cultures, regions, and settings are also recommended.

\section{Conclusions}

PEIP delivered by using a smartphone is effective in improving the parents' knowledge, self-efficacy in symptom management, and parent and child perception of HRQOL. PEIP was innovative in that it targeted all dimensions (physical, emotional, social and cognitive) of HRQOL in children with SCD. The study highlighted the feasibility of using smartphone technology for delivering effective high quality educational interventions. Finally, the family played an important role in the process of care and therefore, developing family-based interventions is the key factor for improving HRQOL in children with SCD. Findings supported the use of PEIP using smartphone technology for improving parental knowledge and parental self-efficacy that led to improvement in the HRQOL in children with SCD. The study also highlighted the effectiveness of smart phone technology for delivering a high quality educational intervention program for parents and their families.

\section{List Of Abbreviations}

SCD : Sickle Cell Disease

HRQOL: Health-Related Quality of Life

PEIP : Parent Educational Intervention Program

SEP: Standard Educational Program

(SCD-PKQ): Sickle Cell Disease Parents Knowledge Questionnaire

\section{Declarations}

Ethical Approval: Ethics approval was obtained from the UCLA Institutional Review Board, the Ministry of Health, the Royal Hospital (RH), and the Sultan Qaboos University Hospital (SQUH). Parents who were interested to participate were provided with a consent form to sign. Also, all children whose parents agreed to participate, agreed to take part of the study and were asked to sign a child assent form. The participants were informed that being part of the research would not affect the care the child receives, and the information would be kept confidential. Moreover, the parents were informed that the participation was voluntary and they had the right to withdraw anytime from the study.

Consent for Publication: The manuscript does not contain identified individuals, videos or images. Children in this study were asked to sign an assent form after their parents' approval.

Availability of Data and Material: All data generated or analyzed during this study are included in the published article.

Competing interests: There is no competing interest to disclose.

Funding: This study was funded by the Ministry of Health, Oman. The role of $\mathrm{MOH}$ was providing fund to design the interventions and develop the educational videos.

Authors' contributions: Al Nasiri Yusra: Written the Literature review, collected study data collection, analyzed and interpreted the patient data, manuscript writing. 
Jacob Eufemia: Edited the literature review, analyzed and interpreted the patient data, edited the manuscript.

Lee Eunince, Nyamathi Adey, Robbins Wendie, Mary-Lynn Brecht, Al Mawali Adhra: Methodology design description, interpreted the data, major contributor in writing the discussion and editing the manuscript.

Acknowledgments: We would like to acknowledge, Sabah Al Kiumi, Maryam Al Shezawi, Dr. Amal Al Subhi, Dr. Nawal Al Maskari, Dr. Karima Al Hanni, Dr. Mohammed Al Hannai, Dr. Muna Al Saddoni, Dr. Yassir Wali, for their valuable contribution in providing content validity for the intervention, translating the study tools, overall all input about the study methodology and facilitating the data collection process in RH \& SQUH.

\section{Authors' information:}

1.Yusra Al Nasiri, Assistant Professor, Oman College of Health Sciences, Oman yusra444@hotmail.com

2.Eufemia Jacob, Associate professor, School of Nursing, University of California Los Angeles, USA ejacob@sonnet.ucla.edu

3.Eunice Lee, Associate professor, School of Nursing, University of California Los Angeles, USA eclee@sonnet.ucla.edu

4.Adey Nyamathi, Founding Dean and Distinguished Professor at Sue \& Bill School of Nursing, University of California Irvine, USA anyamath@uci.edu

5. Mary-Lynn Brecht, Adjunct professor, Statistician, University of California Los Angeles, USA Ibrecht@sonnet.ucla.edu

6.Wendie Robbins, Professor, School of Nursing, University of California Los Angeles, USA wrobbins@sonnet.ucla.edu

7.Adhra Al Mawali, Director of Studies and Research, Ministry of Health, Oman adhra.almawali@gmail.com

\section{References}

1. Ahmed A, Alaskar A, Al-Suliman A, Jazieh A, McClish D, Al Salamah M, Ali Y, Malhan H, Mendoza M, Gorashi A, El-toum M. Health-related quality of life in patients with sickle cell disease in Saudi Arabia. Health Quality of Life Outcomes. 2015; 13(1) : 1-10.

2. Ahn H, Wang W. Sickle cell hepatopathy: clinical presentation, treatment, and outcome in pediatric and adult patients. Pediatric Blood Cancer. 2005 ; 45 :184- 90

3. Ameringer S, Elswick R K, Smith W. Fatigue in Adolescents and Young Adults with Sickle Cell Disease: Biological and Behavioral Correlates and HealthRelated Quality of Life. Journal of Pediatric Oncology Nursing: Official Journal of the Association of Pediatric Oncology Nurses, 31(1): 6-17.

4. Amr M, Amin T, Al-Omair O. Health related quality of life among adolescents with sickle cell disease in Saudi Arabia. The Pan African Medical Journal.2011; 8 (10): 1-15.

5. AL Joauni S, AL Mubayawi, M, Halawa T, AL Mebayawi M. Treatment adherence and quality of life outcomes in patients with Sickle Cell Disease. Saudi Medical Journal. 2013; 34(3): 261- 265

6. Almeida A, Roberts I. Bone involvement in sickle cell disease. British Journal of Haematology, 2005; 129 (4): $482-90$.

7. Al Nasiri Y, Al Mawali A, Jacob E. Identifying Strategies to Help Improve Health-Related Quality of Life in Children With Sickle Cell Disease. International Journal of Current Research. 2017; 9 (12): 62860-62866.

8. American Thoracic Society, Quality of life resources. 2007. http://qol.thoracic.org/sections/key-concepts/functional-status.html. Accessed online March 2018.

9. American Psychological Association. Teaching Tip Sheet: Self-Efficacy. 2016. http://www.apa.org/pi/aids/resources/education/self-efficacy.aspx. Accessed online Jan 2018.

10. Anie K, Egunjobi F, Akinyanju O. Psychosocial impact of sickle cell disorder: perspectives from a Nigerian setting. Globalization and Health. $2010 ; 6: 2-10$.

11. Al-Riyami A , Ebrahim G. Genetics blood disorders survey in the Sultanate of Oman. Journal of Tropical Pediatric. $2003 ; 49$ (1) : 1-20.

12. Badawy S, Thompson A, Lai S, Penedo J, Rychlik K, Liem Health-related quality of life and adherence to hydroxyurea in adolescents and young adults with Sickle Cell Disease. Pediatric Blood and Cancer. 2017; 64 (6): 26-36.

13. Bandura Social Cognitive Theory. In R. Vasta (Ed.), Annals of child development. Vol. 6. Six theories of child development. Greenwich, CT: JAI Press; 1989. p.1-60.

14. Barakat L P, Lutz M., Smith-Whitley, K. Is treatment adherence associated with better quality of life in children with sickle cell disease?. Quality Life Research. 2005; (14): 407-414.

15. Barakat L, Schwartz L, Salamon K, Radcliffe A Family-based Randomized Controlled Trial of Pain Intervention for Adolescents With Sickle Cell Disease. Journal of Pediatric Hematology Oncology. 2010; 32: 540-547.

16. Beverun L, Varni J, Panepinto J. Clinically meaningful interpretation of pediatric health-related quality of life in sickle cell disease. Journal of Pediatric Hematology Oncology. (2014; 37 (2): 128-133.

17. Brousscau D C, Panepinto J, Nimmer M. The number of people with Sickle Cell Disease in the United States: National and state estimates. American Journal of Hematology. 2010; 85 : 77-78.

18. Centers for Disease and Control Prevention. Retrieved from: https://www.cdc.gov/ncbddd/sicklecell/facts.htmlhttps://www.ncbi.nlm.nih.gov/pubmed/17364574. Accessed Jan 2018. 
19. Clay O, Telfair J . Evaluation of a disease-specific self-efficacy instrument in adolescents with sickle cell disease and its relationship to adjustment. Child Neuropsychology, 2007; 13 (2): 188-203.

20. Constantinou C, Payne N, Inusa B. Assessing the quality of life of children with sickle cell anaemia using self-, parent-proxy, and health care professionalproxy reports. British Journal of Health Psychology. 2015; 20: 290-304.

21. Dale JC, Cochran CJ, Lmswap EJ, Buchanan GR. Health-related Quality of Life in children with Sickle Cell Disease. Journal of Pediatric Health Care. 2011; 25(4): 208-10.

22. Dampier C, LeBeau P, Rhee S, Lieff S, Kesler K, Ballas S, Rogers Z, Wang, W. Health-related quality of life in adults with sickle cell disease (SCD): A report from the comprehensive sickle cell centers clinical trial consortium. American Journal of Hematology. 2011; 86: 203-05.

23. Dobson Outcomes results of self-efficacy in children with sickle disease pain who were trained to use guided imagery. Applied Nurses Research. 2015; 28(4): 384-90.

24. Edwards R, Sarlani E, Wesselmann U, Fillingim Quantitative assessment of experimental pain perception: multiple domains of clinical relevance. Pain. 2005; $11 \mathrm{e} 4$ (1): 315-19.

25. El-Hazmi M, Al-Hazmi A, Warsy Sickle Cell Disease in Middle East Arab Countries. The Indian Journal of Medical Research. 2001 ; 135 (5): 597-610.

26. Ferrans C, Zerwic J, Wilbur J, Larson J. Conceptual Model of Health-Related Quality of Life. Journal of Nursing Scholarship. 2005 ; 37 (4): $336-42$.

27. Fernandes M, Kawachi I, Corrêa-Faria , Pattusi M, Paiva S, Pordeus Caries prevalence and impact on oral health-related quality of life in children with sickle cell disease: cross-sectional study. BioMedical Central Oral Health. 2015; 15 (68): 1-8.

28. Fisak B, Belkin M, Lehe A, Bansal M. The relationship between Health-related Quality of Life, treatment adherence and disease severity in pediatric Sickle Cell Disease samples Child. Care, Health, and Development. 2010; 38 (2): 204-10.

29. Forrester AB, Barton-Gooden A, Pitter C Lindo J M. The lived experiences of adolescents with sickle cell disease in Kingston, Jamaica. International Journal of Qualitative Studies on Health and Well-Being. 2015; 10: 1-9.

30. Frei-Jones M, Field J, DeBaun M. Multi-Modal Intervention and Prospective Implementation of Standardized Sickle Cell Pain Admission Orders Reduces 30-Day Readmission Rate. Pediatric Blood Cancer. 2009; 53: 401-05.

31. Hazzard A, Celano M, Collins M, Markov Y. Effects of STARBRIGHT World on Knowledge, Social Support, and Coping in Hospitalized Children With Sickle Cell Disease and Asthma. Children's Health Care. 2002; 31(1): 69-86.

32. Hijmans CT, Fijnvandraat K, Oosterlaan Double disadvantage: A case-control study on health-related quality of life in children with sickle cell disease. Health Quality Life Outcomes. 2010; (8) :117-21.

33. Hines J, Crosby A, Johnson A, Valenzuela J, Kalinyak K, Joiner Engaging Patients With Sickle Cell Disease and Their Families in Disease Education, Research, and Community Awareness. Journal of Prevention \& Intervention in the Community. 2011; 38: 256- 72.

34. Jacob E, Duran J, Stinson J, Lewis M, Zeltzer Remote monitoring of pain and symptoms using wireless technology in children and adolescents with sickle cell disease. Journal of the American Academy of Nurse Practitioners. 2013; 25: 42-54

35. Mahaat G, Scoloveno M, Barnette C, Donnelly Written educational materials for families of chronically ill children. Journal of the American Academy of Nurse Practitioners. 2007; $19: 471-76$.

36. Maakaron Sickle Cell Anemia Clinical Presentation Medscape . 2016. Retrieved online:http://emedicine.medscape.com/article/205926clinical? pa=dANGsu6QLas2jfw6AqwjarL1SXqHBGuZzYFYL7zPyC2RBDa2enwbzwT90jVqKBnj6TB0GpZi\%2Bkj018DEWRkHjabXa1aj0VoWN5\%2BW19QIDeU\%3

37. McClellan B, Schatz C, Sanchez C, Stancil Use of Handheld Wireless Technology for a Home-based Sickle Cell Pain Management. Journal of Pediatric Psychology. 2009; 34 (5): 564-573.

38. Ministry of Health. National genetic blood disorders survey. Second Edition, Bahrain :Awal Bress, 2010.

39. Miller A, Gladwin Pulmonary complications of sickle cell disease. American Journal of Respiratory Critical Care Medicine. $2012 ; 185$ (11): $1154-65$.

40. National, Heart, Lung and Blood Institute. https://www.nhlbi.nih.gov/health-topics/sickle-cell-disease . Accessed on Jan , 2018.

41. Nwenyi E, Leafman J, Mathieson K, Ezeobah N. Differences in quality of life between paediatric sickle cell disease who use hydroxuria and who do not. International Journal of Health Care Quality Assurance. 2013; 27 (6): 468-81

42. Oman, (2014). Effort to combat blood disorders in Omanis. Accessed online via: http://timesofoman.com/article/30676/Oman/Effort-to-combat-blooddisorders-in-Omanisdisqussion-0disqussion-0disqussion-0disqussion-0disqussion-0disqussion-0disqussion-0disqussion-0disqussion-0disqussion-0

43. Palermo T, Valenzuela D, Stork A randomized trial of electronic versus paper pain diaries in children: Impact on compliance, accuracy, and acceptability. Pain. 2004; 107 (3):213- 19

44. Panipento JA, Torres S, Varni J W. Development of the PedQL sickle cell disease module items: Qualitative methods. Quality Life Research. 2012; (21): 341-52

45. Panepinto JA, Torres S, Bendo C B. PedsQLt Sickle Cell Disease Module: feasibility, reliability, and validity. Pediatric Blood Cancer. $2013 ; 60$ : $1338-44$.

46. Panipento J A, O'Mahar M, DeBaun M. Health-related quality of life in children with sickle cell disease: Child and parents perception. Journal of Pediatric Hematology. 2005; (130): 437-44

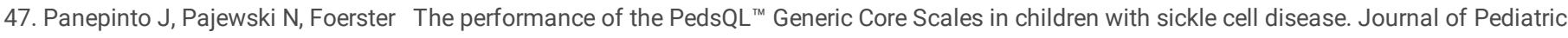
Hematology Oncology. 2008; 30, 666-73

48. Panepinto J, Torres S, Bendo PedsQL ${ }^{T M}$ Sickle Cell Disease Module: Feasibility, reliability, and validity. Pediatric Blood Cancer. 2013; 60: $1338-44$.

49. Rajab A, Patton M \& Modell Study of hemoglobinopathies in Oman through a national register. Saudi Medical Journal. $2000 ; 21$ (12): $1168-72$.

Page $11 / 15$ 
50. Reagan M, DeBaun M, Frei-Jones Multi-Modal Intervention for the Inpatient Management of Sickle Cell Pain Significantly Decreases the Rate of Acute Chest Syndrome. Pediatric Blood Cancer. 2011; 56: 262-66.

51. Shahine R. Kurdahi L, Karam D, Abboud Educational Intervention \to Improve the Health Outcomes of Children With Sickle Cell Disease. Journal of Pediatric Health Care. 2015; 29 (1): 54-60.

52. Sickle Cell Association of America. https://www.sicklecelldisease.org/\#. Accessed March, 2017.

53. Smith K, Patterson C, Szabo M, Barakat, M. Predictors of Academic Achievement for School Age Children with Sickle Cell Disease. Advances in School Mental Health Promotion. 2013; 6(1): 5-20.

54. Sousa K, Kwok O. Putting Wilson and Cleary to the test: analysis of a HRQOL conceptual model using structural equation modeling. Quality of Life Research. 2006; 15, 725: 37.

55. The Internet Stroke Centre. http://www.strokecenter.org/patients/about-stroke/pediatric-stroke/stroke-as-a-complication-of-sickle-cell-disease/. Accessed Jan, 2018.

56. Varni J, Seid M, Kurtin P. PedsQL ${ }^{T M} 4.0$ : Reliability and validity of the pediatric quality of life inventory version 4.0 generic core scales in healthy and patient populations. Med Care. 2003; 39, 800-12.

57. Wilson I, Cleary P. Linking clinical variables with health-related quality of life: A conceptual model of patient outcomes. Journal of American Medical Association. 1995; 273: 59-65.

58. World Health Organization. Sickle Cell Disease. . https://www.uptodate.com/contents/sickle-cell-disease-in-sub-saharan-africa/print Accessed Jan, 2018.

59. Wrotriak B, Schall J, Brault M, Balmer D, Stallings V. Health-Related Quality of Life in Children With Sickle Cell Disease Using the Child Health Questionnaire. Journal of Pediatric Health Care. 2012; 28 (1) : 14 - 22.

60. Zempsky W T, Palermo T M, Corsi J M, Lewandowski AS, Zhou C, Casella J F. Daily changes in pain, mood and physical function in youth hospitalized for sickle cell disease pain. Pain Research \& Management: The Journal of the Canadian Pain Society. 2013; 18 (1), 33-38.

\section{Table}

Table 1. Demographics $(\mathrm{N}=72)$

\begin{tabular}{|c|c|c|}
\hline & PEIP (N=37) & $\operatorname{SEP}(\mathbf{N}=35)$ \\
\hline \multicolumn{3}{|l|}{ Parents } \\
\hline Age & $39 \pm 4.12$ & $40 \pm 6.01$ \\
\hline \multicolumn{3}{|l|}{ Means (SD) } \\
\hline \multicolumn{3}{|l|}{ Sex } \\
\hline Male & $12(32 \%)$ & $24(69 \%)$ \\
\hline Female & $25(68 \%)$ & $11(31 \%)$ \\
\hline \multicolumn{3}{|l|}{ Marital Status } \\
\hline Married & 35 (95\%) & $35(100 \%)$ \\
\hline Divorced & $2(5 \%)$ & 0 \\
\hline \multicolumn{3}{|l|}{ Education } \\
\hline$\leq$ High School & $24(64 \%)$ & $26(75 \%)$ \\
\hline Associate (AD) & 7 (19\%) & $4(11 \%)$ \\
\hline Bachelor (BS/BA) & $5(14 \%)$ & $4(11 \%)$ \\
\hline Master (MS/MA) & $1 \quad(3 \%)$ & $1 \quad(3 \%)$ \\
\hline \multicolumn{3}{|l|}{ Children } \\
\hline \multicolumn{3}{|l|}{ Age } \\
\hline Means (SD) in years & $10 \pm 1.3$ & $10 \pm 1.2$ \\
\hline \multicolumn{3}{|l|}{ Sex } \\
\hline Male & 19 (51\%) & $15(43 \%)$ \\
\hline Female & $18(49 \%)$ & $20(57 \%)$ \\
\hline Hydroxyurea & 15 (41\%) & $18(49 \%)$ \\
\hline
\end{tabular}

\section{Figures}




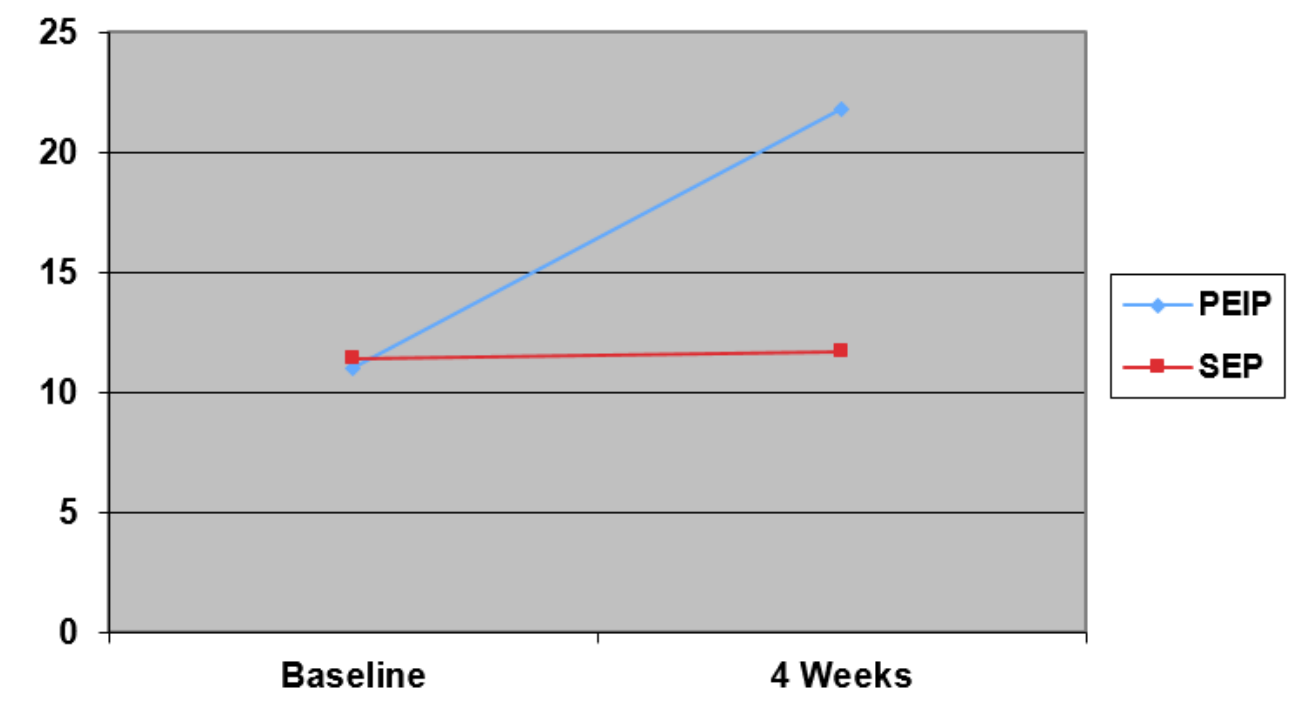

**Within: $F=23.14, \eta 2=.4, p=.001$; Between: $F=477.9, \eta 2=.87, p=.001$. The PEIP group had a significant improvement in the knowledge scores at 4 weeks, compared to baseline. There were significant differences in the knowledge scores $(p=0.001)$ between the $P E I P$ and SEP at 4 weeks.

Figure 1

Knowledge Scores at Baseline and 4 Weeks in PEIP \& SEP Groups

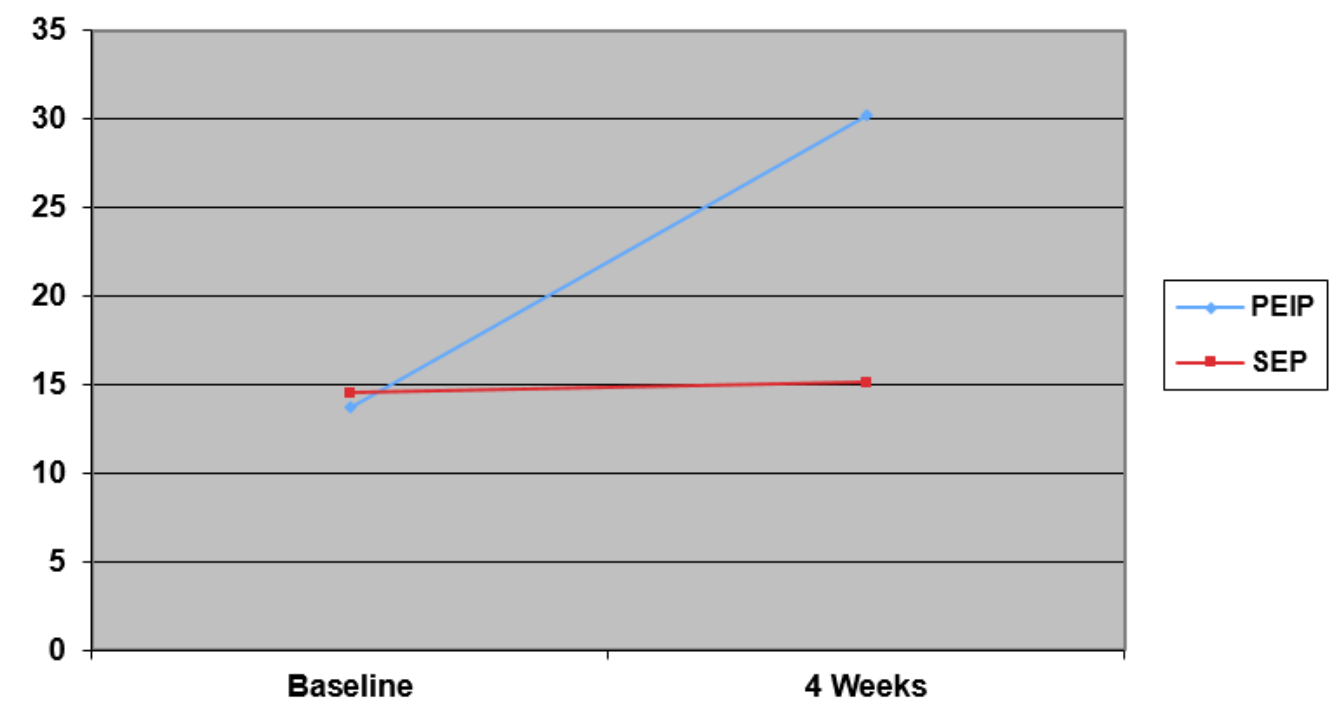

\footnotetext{
**Within: $F=12.4, \eta 2=.3, p=0.001$; Between: $F=666.2, \eta 2=.91, p=0.001$. The PEIP group had a significant improvement in the self efficacy scores at 4 weeks, compared to baseline. There were significant differences in the self-efficacy scores $(p=0.001)$ between the PEIP and SEP at 4 weeks.
}

Figure 2

Self-Efficacy Scores at Baseline and 4 Weeks in PEIP \& SEP Groups 


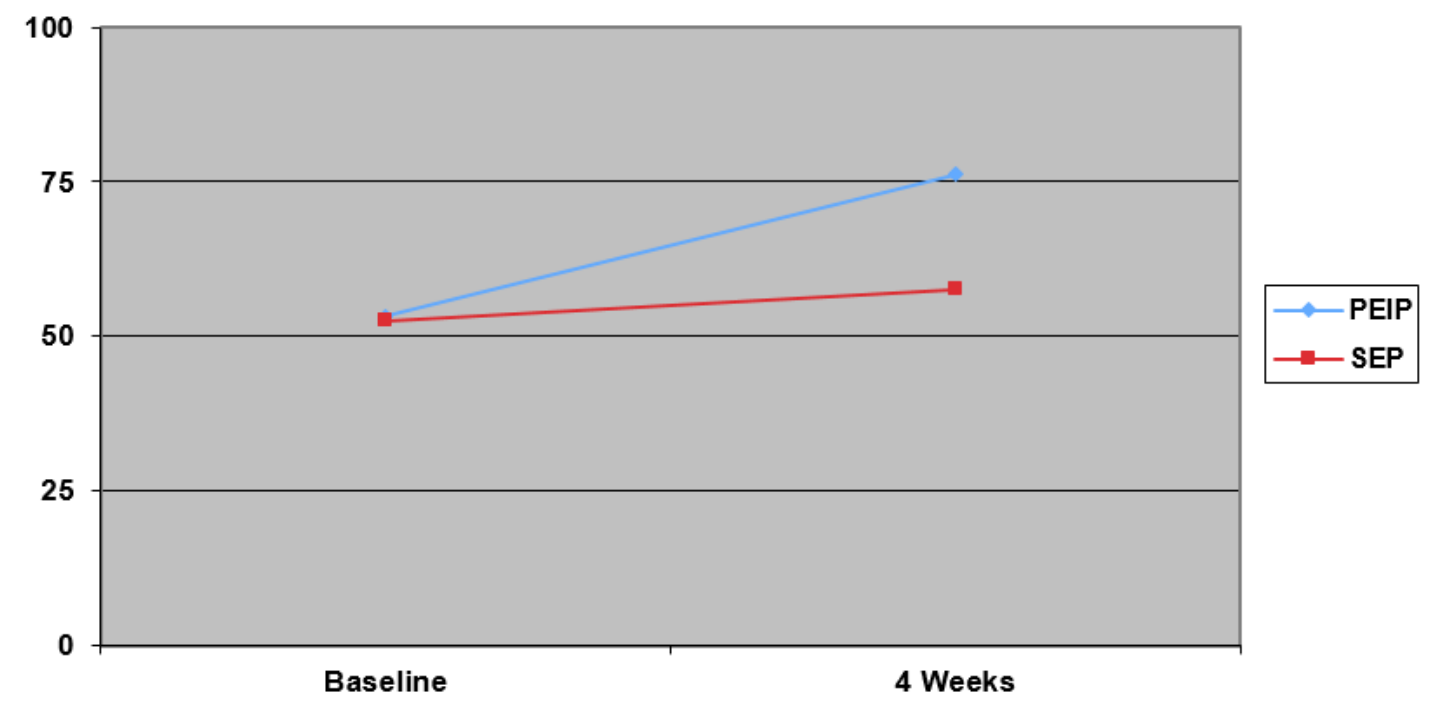

**Within: $F=10.91, \eta 2=.3, p=.001$; Between: $F=148.92, \eta 2=.7, p=.001$. The PEIP group had a significant improvement in the HRQOL-SCD scores at 4 weeks, compared to baseline. There were significant differences in the HRQOL scores $(p=.001)$ between the PEIP and SEP at 4 weeks.

Figure 3

HRQOL-SCD Scores at Baseline and 4 Weeks in PEIP \& SEP Groups

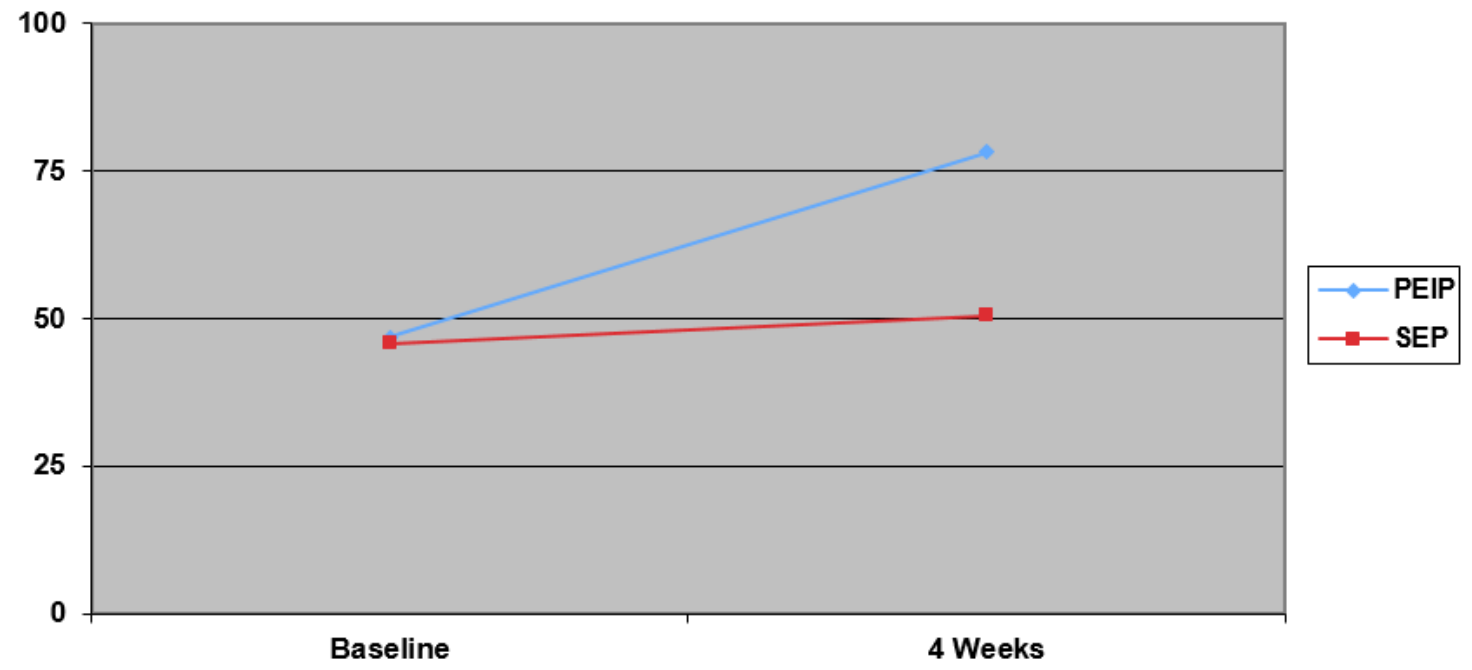

**Within: $F=13.16, \eta 2=.3, p=.001$; Between: $F=317.26, \eta 2=.8, p=.001$. The PEIP group had a significant improvement in the self efficacy scores at 4 weeks, compared to baseline. There were significant differences in the self-efficacy scores $(p=.001)$ between the PEIP and SEP at 4 weeks

\section{Figure 4}

PedsQL-GENERIC Scores at Baseline and 4 Weeks in PEIP \& SEP groups 


\section{CONSORT Flow Diagram}

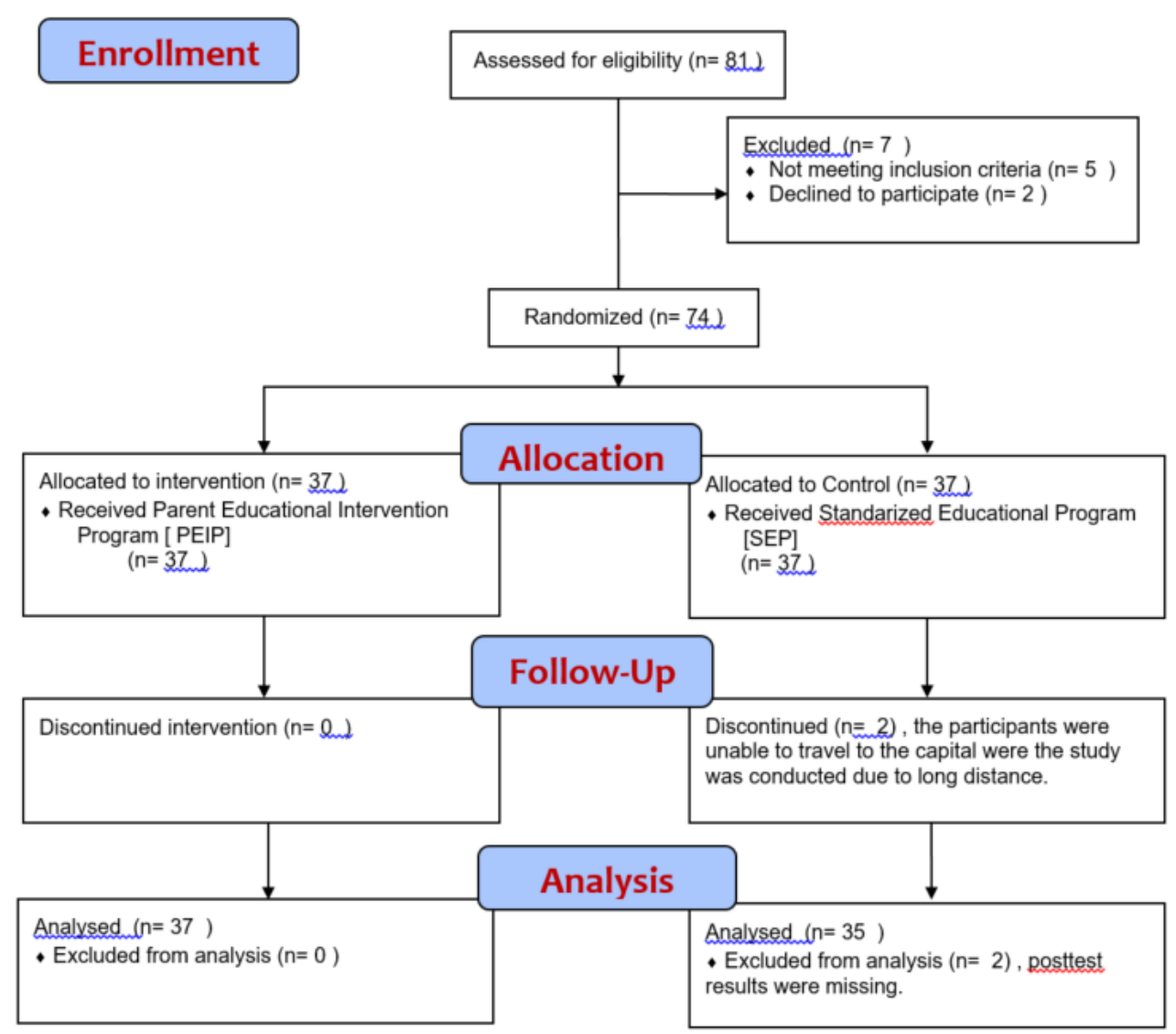

Figure 5

Consort Flow Diagram for PEIP \& SEP

\section{Supplementary Files}

This is a list of supplementary files associated with this preprint. Click to download.

- additionalfile.docx 